\title{
Investigating associations between child bone mineral density and vitamin D status, diet, physical activity, and body composition at 5 years of age - Findings from the ROLO Kids Study
}

\author{
MK McVey ${ }^{1}$, AA Geraghty ${ }^{1}$, EC O’Brien $^{1}$, MJ McKenna $^{1,2,3}$, MT Kilbane ${ }^{4}$, RK Crowley ${ }^{2,3}$, \\ PJ Twomey ${ }^{3,4}$ and FM McAuliffe ${ }^{1}$ \\ ${ }^{1}$ UCD Perinatal Research Centre, Obstetrics \& Gynaecology, School of Medicine, National Maternity Hospital, \\ University College Dublin, Dublin, Ireland, ${ }^{2}$ Department of Endocrinology, St. Vincent's University Hospital, \\ Dublin, Ireland, ${ }^{3}$ School of Medicine, University College Dublin, Dublin, Ireland and ${ }^{4}$ Department of Clinical \\ Chemistry, St. Vincent's University Hospital, Dublin, Ireland
}

Bone health is extremely important in early childhood because children with low bone mineral density (BMD) are at a greater risk of bone fractures $^{(1)}$. Physical activity is beneficial for bone health in adolescents and body composition has also been associated with BMD during teenage years ${ }^{(2,3)}$. While adequate intake of both calcium and vitamin $\mathrm{D}$ are important for bone health in pre-pubescent children, there is limited research on the determinants of good bone health in early childhood.

Whole body BMD was measured by dual-energy X-ray absorptiometry (DXA) in 102 children from the ROLO Kids Study. Physical and sedentary activity levels were measured using the Children's Leisure and Activities Study Survey (CLASS). Dietary intakes were measured using a food frequency questionnaire, which were both completed by the mother. Child weight, height, circumferences and skinfolds were measured by the research team. Blood samples were drawn for measurement of circulating serum 25-hydroxyvitamin D (25OHD) concentrations. Statistical analysis was carried out using Pearson and Spearman correlations with multiple linear regression analysis.

There was no association between physical activity, sedentary behaviour, dietary calcium, dietary vitamin D, or 25OHD with BMD. Body composition was significantly associated with BMD; weight, body mass index, arm circumference and chest circumference were positively associated with BMD $(\mathrm{p}<0.01)$ as was abdomen, hip and thigh circumference $(<0.05)$. Length also correlated positively with BMD $(\mathrm{p}<0 \cdot 012)$.

Table 1. Bone Mineral Density at 5 years of Age Based on Current Health Recommendations

\begin{tabular}{|c|c|c|c|c|c|}
\hline \multirow[b]{2}{*}{ Recommendations } & \multicolumn{2}{|c|}{$\begin{array}{c}\text { Meeting } \\
\text { Recommendation } \\
\end{array}$} & \multicolumn{2}{|c|}{$\begin{array}{c}\text { Not Meeting } \\
\text { Recommendation } \\
\end{array}$} & \multirow[b]{2}{*}{$P$} \\
\hline & $\overline{\mathrm{N}}$ & $\overline{\mathrm{BMD}}$ & $\mathrm{N}$ & BMD & \\
\hline Dietary Calcium Intake $(>800 \mathrm{mg})^{1}$ & 46 & $0 \cdot 674$ & 51 & $0 \cdot 674$ & 0.94 \\
\hline Physical Activity Level (>400mins/week) ${ }^{2}$ & 50 & 0.678 & 23 & $0 \cdot 675$ & 0.79 \\
\hline Screen time Level $(<1 \text { hour/day })^{3}$ & 19 & $0 \cdot 663$ & 53 & 0.679 & $0 \cdot 17$ \\
\hline
\end{tabular}

BMD: Bone Mineral Density (cm/g). Significant at $P<0.05$

Recommendations based on (1) Scientific Guidelines for Healthy Eating in Ireland (2011), (2) The National Guidelines on Physical Activity for Ireland (2009). (3) Media Use in School-Aged Children and Adolescents: AAP (2016)

We found no association between self-reported lifestyle and dietary factors with bone health in early childhood as measured by BMD analysis using DXA. Increased body size was linked with higher bone mineral density. This is important for bone health in later life as optimizing BMD during childhood may reduce the risk of fractures in adolescence and adult life.

1. Clark EM, Ness AR, et al. (2006) J Bone Miner Res 21(9), 1489-1495.

2. Hind K, Burrows M (2007) Bone 40(1), 14-27.

3. Clark EM, Ness AR et al. (2006) J Clin Endocrinol Metab 91(7), 2534-2541. 